\title{
Dual-stage nanopositioning for high-speed scanning probe microscopy
}

\section{Journal Article}

\section{Author(s):}

Tuma, Tomas; Haeberle, Walter; Rothuizen, Hugo; Lygeros, John (D); Pantazi, Angeliki; Sebastian, Abu

\section{Publication date:}

2014-06

\section{Permanent link:}

https://doi.org/10.3929/ethz-b-000096540

Rights / license:

In Copyright - Non-Commercial Use Permitted

Originally published in:

IEEE/ASME Transactions on Mechatronics 19(3), https://doi.org/10.1109/TMECH.2013.2266481 


\title{
Dual-stage nanopositioning for high-speed scanning probe microscopy
}

\author{
Tomas Tuma, Walter Haeberle, Hugo Rothuizen, John Lygeros, Angeliki Pantazi and Abu Sebastian
}

\begin{abstract}
This paper presents a dual-stage approach to nanopositioning in which the trade-off between scanner speed and range is addressed by combining a slow, large-range scanner with a short-range scanner optimized for high-speed, highresolution positioning. We present the design, finite-element simulations and experimental characterization of a fast custombuilt short-range scanner. The short-range scanner is based on electromagnetic actuation to provide high linearity, has a clean, high-bandwidth dynamical response and is equipped with a low-noise magnetoresistance-based sensor. By using advanced noise-resilient feedback controllers, the dual-stage system allows large-range positioning with subnanometer closed-loop resolution over a wide bandwidth. Experimental results are presented in which the dual-stage scanner system is used for imaging in a custom-built atomic force microscope.
\end{abstract}

\section{INTRODUCTION}

Precise positioning on the nanometer scale, often referred to as nanopositioning, is a key enabling technology for nanoscale science and engineering. With a vast range of applications across various fields such as information technology [1], semiconductor manufacturing [2] and biology [3], nanopositioning has become an important interdisciplinary research topic interconnecting the fields of control, mechanical and electrical engineering.

One of the key techniques in nanotechnology that critically relies on nanopositioning is scanning probe microscopy (SPM) [4]. SPM allows the interrogation and interaction with matter down to the atomic level by probing surfaces with an atomically sharp probe tip. For instance, in atomic force microscopy (AFM), which is a type of SPM, the probe is positioned relative to the sample by means of a nanopositioner (often called a scanner), and the properties of the sample surface are inferred from the forces acting on the probe tip as it traverses the sample.

Significant research efforts have been devoted to the design and control of SPM scanners [5]. Piezoelectric tube scanners were first introduced for scanning tunneling microscopy in 1986 [6] and have been used extensively in SPM [7], [8], [9] owing to their high resolution and low cost. Their disadvantage is the low resonant frequencies and a relatively large amount of cross-coupling. In another approach, piezoactuated flexure-guided scanners with serial or parallel kinematics are used, which can reach a high actuation bandwidth and at the same time, retain excellent mechanical properties [10], [11], [12], [13].

T. Tuma, W. Haeberle, H. Rothuizen, A. Pantazi and A. Sebastian are with IBM Research - Zurich, 8803 Rueschlikon, Switzerland \{uma, wha, rth, agp, ase\}@ zurich.ibm.com

T. Tuma and J. Lygeros are with Automatic Control Laboratory, Swiss Federal Institute of Technology, 8092 Zurich, Switzerland lygerosecontrol.ee.ethz.ch
To achieve high positioning precision over a high bandwidth, SPM scanners are typically operated under feedback control. Feedback control is essential to compensate for environmental disturbances such as building vibrations and temperature fluctuations, and also for fabrication uncertainties, mechanical resonances and nonlinearities of the actuator. The closed-loop resolution, determined by the closed-loop bandwidth and the sensor characteristics, is of critical importance as it governs the overall resolution of the SPM. In high-speed SPM, the closed-loop bandwidth is typically large which leads to an increase in the amount of measurement noise that enters the feedback loop and deteriorates the resolution. Even if high-end noncontact sensors are used for position feedback, the standard deviation of the position measurement signal amounts to several nanometers over the closed-loop bandwidth.

This paper presents the design and characterization of a dual-stage nanopositioning system specifically tailored for high-resolution sub-nanometer positioning at high speeds. To that end, the nanopositioning system is designed to meet three fundamental requirements. First, high open-loop bandwidth is required to enable both high-speed and highresolution operation. Allowing low open-loop bandwidth would deteriorate the closed-loop resolution as a broadband control effort would be required to obtain a flat closedloop frequency response over a wide bandwidth. Second, well-defined dynamics and high linearity are required, such that the scanner can be modeled accurately and used with advanced noise-resilient feedback controllers, such as resonant control [14], feedback controllers with a shaped noisesensitivity transfer function [15], signal transformation [16] and impulsive control [17]. Third, a large scan range is required; the bandwidth and linearity should not be obtained at the expense of the scan range.

Conventional SPM scanners based on the piezoelectric actuation principle, such as piezoelectric tube scanners and piezo-actuated flexure stages, do not satisfy the above requirements owing to the inherent nonlinear characteristics of piezoelectric actuators, such as drift, creep and hysteresis [18]. To compensate for these nonlinearities, feedback control over a wide bandwidth is typically required, which negatively affects the positioning resolution. To circumvent these problems, our scanner design is based on electromagnetic actuation which has been widely used in applications such as in hard-disk magnetic recording [19], probe-based data storage [20], [21] tape storage [22] and semiconductor lithography [23]. The advantage of electromagnetic actuation is its high linearity; on the other hand, electromagnetic actuators typically provide only limited actuation force, which has to be reflected in the scanner design. 


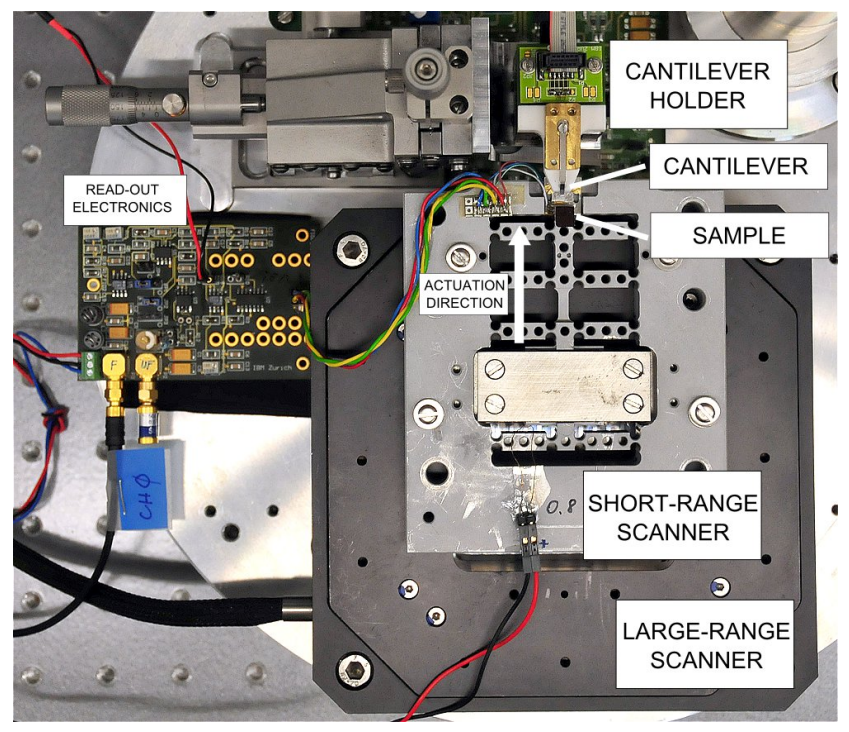

Fig. 1. Photograph of the dual-stage scanner system, showing the singleaxis short-range scanner mounted on the three-axis large-range positioning stage.

To illustrate a fundamental trade-off involved in the scanner design, let us assume that the dynamical response of the scanner resonant modes can be modeled as that of a harmonic oscillator. A simple harmonic oscillator with mass $m$ and stiffness $k$ has the resonant frequency

$$
\omega=\sqrt{\frac{k}{m}} .
$$

Hence, to obtain a high open-loop bandwidth, the mass of the scanner should be minimized and the stiffness maximized. However, the extent to which the mass can be minimized is limited because of the manufacturing process as well as the ability to mount samples with variable mass. On the other hand, increasing the stiffness of the scanner conflicts with achieving large scan ranges with the limited actuation force of the electromagnetic actuator. This is a fundamental design trade-off which renders the above requirements challenging if not impossible to achieve in a conventional scanner design.

This paper presents a novel approach based on decoupling the requirements on the speed and the range of the scanner, an idea central to the dual-stage positioning concept as known, for example, in the field of optical recording [24], hard-disk magnetic recording [25], robotics [26], [27] and micromanipulation [28]. In SPM, the dual-stage concept was introduced in vertical positioning [29], [30], but so far, only little research effort has been devoted towards dual-stage lateral nanopositioning.

In the dual-stage nanopositioning system introduced in this paper, two serially coupled scanners are used: a first-stage scanner for large-range positioning with a low bandwidth (large-range scanner) and a second-stage scanner for shortrange positioning with a high bandwidth (short-range scanner). The large-range scanner is a commercial piezo-actuated stage with three-degrees-of-freedom displacement capability whereas the electromagnetically actuated short-range scanner is custom-designed for high-speed operation along a single

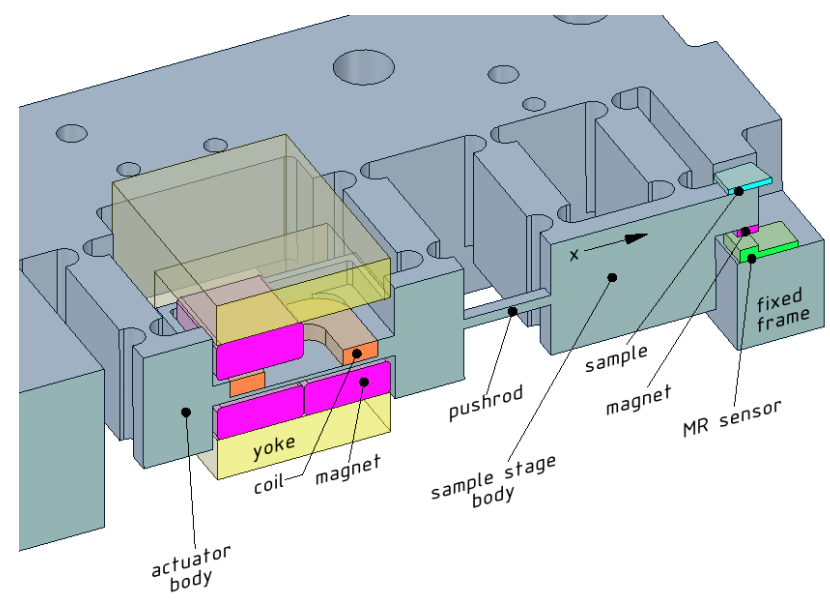

Fig. 2. Half-symmetry crosssection of the short-range scanner. The lightening holes and one of the magnets have been omitted in this view for clarity.

axis. This configuration is particularly suitable for the widely used raster-scanning SPM, but can also be used for more recent scanning modes such as feature-tracking [31]. The prototype system demonstrated in this paper achieves realtime video-rate SPM imaging with nanometer-scale resolution and speed of more than 1 frame/s, while having a range of $100 \mu \mathrm{m}$ which is above most state-of-the-art SPM flexurebased nanopositioners (a recent comparison can be found e.g. in [12]). The work is based on the preliminary results published in [32] and [33].

The paper is organized as follows. In Section II, we present the mechanical design, finite-element simulations and modeling of the custom-designed short-range scanner. In Section III, the experimental characterization of the scanner is presented, including measurements of the scanner range, dynamic response and thermo-mechanical coupling. Section IV presents a noise-resilient feedback control design for the short-range scanner. Section V presents experimental results in which the dual-stage system is used for high-speed and large-range imaging in a custom-built AFM. Section VI concludes the paper.

\section{SCANNER DESIGN}

Because most nanopositioning applications are of the raster-scan type, the more demanding trade-offs between positioning range, bandwidth, and accuracy apply to only one axis. By using a custom-built short-range scanner, our dual-stage approach improves the performance, for this one axis, in the high-frequency portion of the dynamic range, while retaining the larger stroke of the slower in-plane axis. Figure 1 shows a photograph of the overall prototype scanner system. Slow, large-range positioning for all axes is provided by a commercial 3-axis piezo-actuated positioner (model PI517 from Physik Instrumente), with stroke ranges of $100 \mu \mathrm{m}$ for the in-plane directions $x$ and $y$, and $20 \mu \mathrm{m}$ for $z$ out-of plane. The custom-built short-range scanner is mounted directly on the large-range scanner and is specifically designed for high speed, high linearity and clean dynamical behavior 

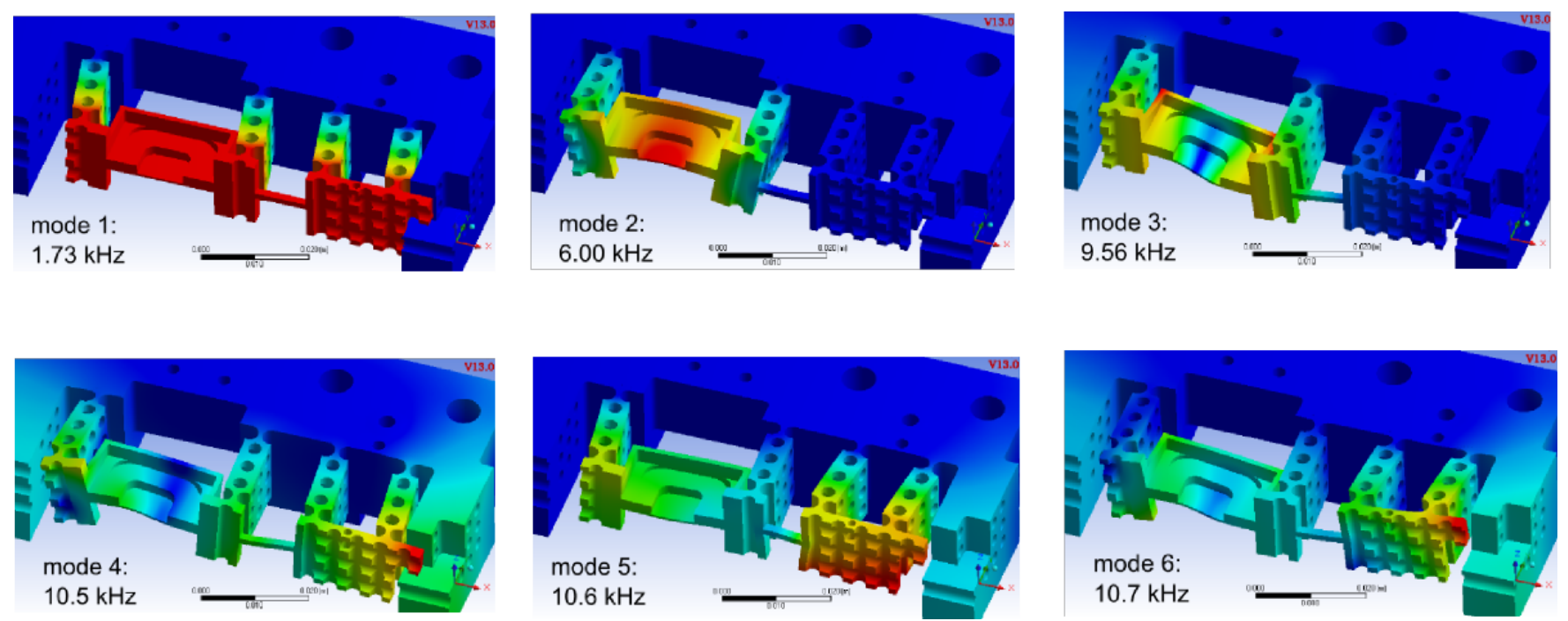

Fig. 4. Eigenmode shapes from finite element modal analysis. Only the half-symmetry cross-section is shown in the figure for illustration purposes, however, the simulation was done for the complete system.

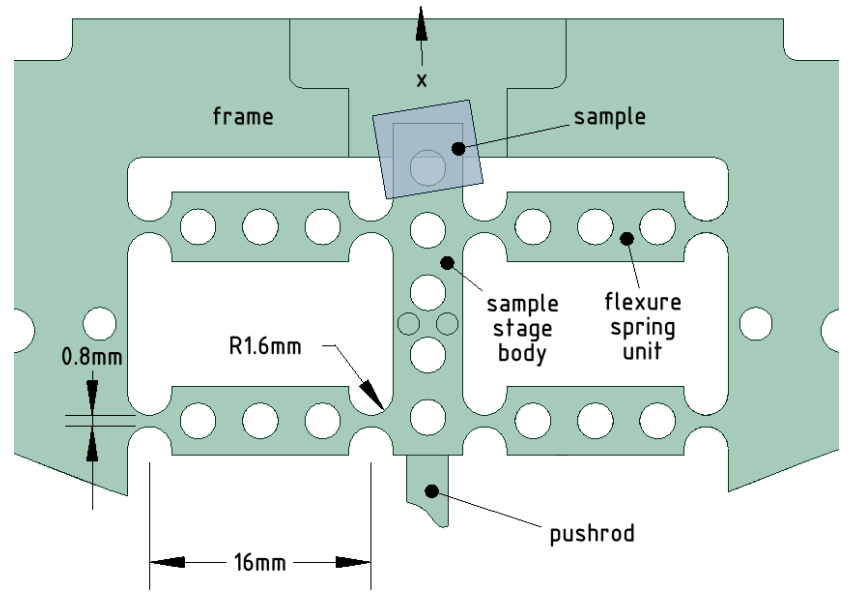

Fig. 3. Detail of the suspension spring of the short-range scanner with principal dimensions.

in the fast positioning axis. In the following, the mechanical design of the short-range scanner is described in detail, along with a model of the dynamics in the actuation direction and a built-in high-bandwidth position sensor.

\section{A. Design considerations}

The custom-built short-range scanner for high-speed positioning comprises two rigidly linked moving bodies, a sample-carrying table and an actuator sub-assembly, each of which is guided uniaxially by a set of profiled flexures. A cross-sectional schematic of the short-range scanner, including actuation and position sensing components discussed in Section II-D, is shown in Figure 2.

The suspension of each of the moving parts is based on four high-aspect-ratio flexure spring units arranged in symmetrical pairs on either side of the moving bodies, as shown in a cut-away view in Figure 3. Each flexure unit is shaped with two circular notch hinges for a well-controlled location of bending and ease of fabrication. Having found single-side suspension inadequate in preliminary simulations, due to excessive run-out and compliance in the out-of-plane direction, we opted for this doubly clamped configuration which provides much better cross-talk performance [12], [34]. Although the doubly clamped boundary conditions do lead to stress-stiffening in principle, the relative length of the flexures (16 $\mathrm{mm}$ between the circular notch hinges) compared to the small displacement range required (on the order of $+/-1 \mu \mathrm{m}$ ) makes these nonlinearities negligible.

We use electromagnetic actuation over the more conventional choice of a piezo stack in order to ensure linearity, and in particular to avoid the latter's hysteretic behavior. The actuator consists of a racetrack-shaped flat coil located on the rotor (moving) side, and an arrangement of four $\mathrm{NdFeB}$ magnets on the stator side, which immerse the long sides of the coil in a uniform magnetic field and generate Lorentz forces on the coil winds along the intended direction of motion $(x)$. The measured force gain of this actuator is approximately 0.9 N/A. The actuator is driven by a low-noise voltage-to-current amplifier with gain of $0.08 \mathrm{~A} / \mathrm{V}$. In the control design and experiments presented in the remainder of this paper, the voltage-to-current amplifier is considered a part of the actuator for brevity.

Decoupling of the two moving bodies via the small-section $\left(1.2 \times 3.0 \mathrm{~mm}^{2}\right)$ connection rod serves two purposes: first, to mechanically attenuate the effects of any residual out-ofplane forces exercised by the actuator on the sample table, and secondly, to lengthen the thermal time constant of heatup of the sample table subassembly when the actuator is run at high power or low duty cycle. Lastly, we have perforated all moving parts with lightening holes in order to decrease the carried mass, and thereby make the stage faster.

\section{B. Finite-element simulations}

A summary of the eigenmode shapes obtained in a modal analysis using the commercial tool for finite element analysis 


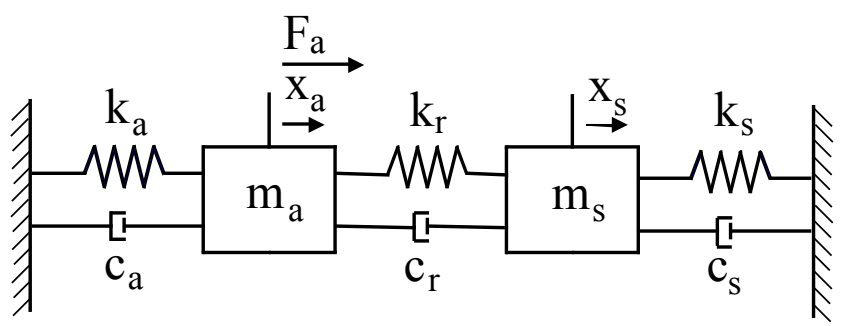

Fig. 5. Free-body diagram of a two-mass model of the short-range scanner.

ANSYS is shown in Figure 4. The principle eigenmodes of interest are the fundamental mode of the suspension system, corresponding to intended actuation along the fast axis, and mode $\mathrm{Nr}$. 5, which corresponds to elongation/ compression of the connection rod along its long axis due to finite material stiffness. Other eigenmodes in the intermediate frequency range involve bending of the coil-holding frame, and are not expected to show up in a significant way in the dynamics unless the actuation components are misaligned so as to generate a significant out-of-plane force or bending moment.

\section{Model}

The short-range scanner was specifically designed with the objective of clean dynamical behavior over the whole actuation bandwidth in order to simplify the design, modeling and feedback control of the scanner. To that end, the scanner was designed such that its dynamics in the actuation direction correspond to an interconnection of harmonic oscillators.

The dynamics in the actuation direction can be accurately modeled as a spring-mass-damper system with two masses connected by spring-damper pairs as shown in the free-body diagram in Figure 5. The masses $m_{a}$ and $m_{s}$ correspond to the actuator body and the scan table body, respectively. These two masses are connected by the small-section connection rod which is modeled as a spring-damper system with stiffness $k_{r}$ and damping coefficient $c_{r}$, respectively, to capture effects related to its finite stiffness at high frequencies. The connection of the actuator and the scan table body to the mechanical ground is also modeled with spring-damper pairs with coefficients $k_{a}$ and $c_{a}$ for the actuator side and $k_{s}$ and $c_{s}$ for the scan table side, respectively.

By using Newton's laws of motion, the equations governing the scanner motion are

$$
\begin{aligned}
F_{a}(t)= & m_{a} \ddot{x_{a}}(t)+ \\
& +k_{a} x_{a}(t)+c_{a} \dot{x_{a}}(t)+ \\
& +k_{r}\left(x_{a}(t)-x_{s}(t)\right)+ \\
& +c_{r}\left(\dot{x_{a}}(t)-\dot{x_{s}}(t)\right) \\
0= & m_{s} \ddot{x_{s}}(t)+ \\
& +k_{s} x_{s}(t)+c_{s} \dot{x_{s}}(t)+ \\
& +k_{r}\left(x_{s}(t)-x_{a}(t)\right)+ \\
& +c_{r}\left(\dot{x_{s}}(t)-\dot{x_{a}}(t)\right)
\end{aligned}
$$

Here, $x_{a}$ and $x_{s}$ denote the position of the actuator and scan table body, respectively, and $F_{a}$ specifies the actuation
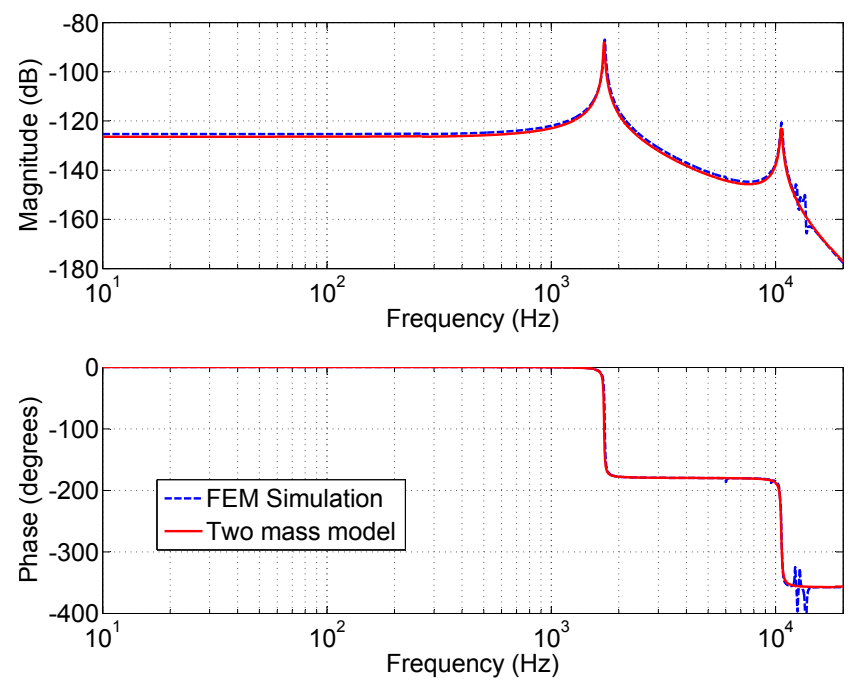

Fig. 6. Estimated frequency response between the actuation signal and the displacement of the scan table in the actuation direction. The figure compares the response obtained from finite element simulation to that computed using the two-mass model.

TABLE I

COEFFICIENTS USED IN THE TWO-MASS MODEL OF THE SHORT-RANGE SCANNER

\begin{tabular}{ll||ll||ll}
\hline \multicolumn{1}{l|}{ Actuator } & & Scan table & & Rod & \\
\hline \hline$m_{a}(\mathrm{~kg})$ & 0.0126 & $m_{s}(\mathrm{~kg})$ & 0.002 & $k_{r}(\mathrm{~N} / \mathrm{m})$ & $7 \times 10^{6}$ \\
$k_{a}(\mathrm{~N} / \mathrm{m})$ & $10^{6}$ & $k_{s}(\mathrm{~N} / \mathrm{m})$ & $10^{6}$ & $c_{r}$ & 0.8 \\
$c_{a}$ & 0.8 & $c_{s}$ & 0.8 & & \\
\hline
\end{tabular}

force exerted on the actuator body. Using the above equations, the motion of the scan table, $y(t)$, in response to the actuation, $u(t)$, can be modeled as the state-space system

$$
\begin{aligned}
\dot{x}(t) & =A x(t)+B u(t) \\
y(t) & =C x(t)
\end{aligned}
$$

with the four-component state

$$
x(t)=\left[\begin{array}{llll}
x_{a}(t) & \dot{x_{a}}(t) & x_{s}(t) & \dot{x_{s}}(t)
\end{array}\right],
$$

the dynamics matrix

$$
A=\left[\begin{array}{cccc}
0 & 1 & 0 & 0 \\
-\frac{k_{a}+k_{r}}{m_{a}} & -\frac{c_{a}+c_{r}}{m_{a}} & \frac{k_{r}}{m_{a}} & \frac{c_{r}}{m_{a}} \\
0 & 0 & 0 & 1 \\
\frac{k_{r}}{m_{s}} & \frac{c_{r}}{m_{s}} & -\frac{k_{r}+k_{s}}{m_{s}} & -\frac{c_{r}+c_{s}}{m_{s}}
\end{array}\right]
$$

the input matrix

$$
B=\left[\begin{array}{llll}
0 & 1 / m_{a} & 0 & 0
\end{array}\right]
$$

and the output matrix

$$
C=\left[\begin{array}{llll}
0 & 0 & 1 & 0
\end{array}\right] .
$$

Figure 6 compares the transfer function between the actuation force, $F_{a}(t)$, and the scan table displacement, $x_{s}(t)$, as calculated using the finite element simulations and using the above 4-state state space model. As expected from the eigenmode analysis, the dynamics are very clean with 


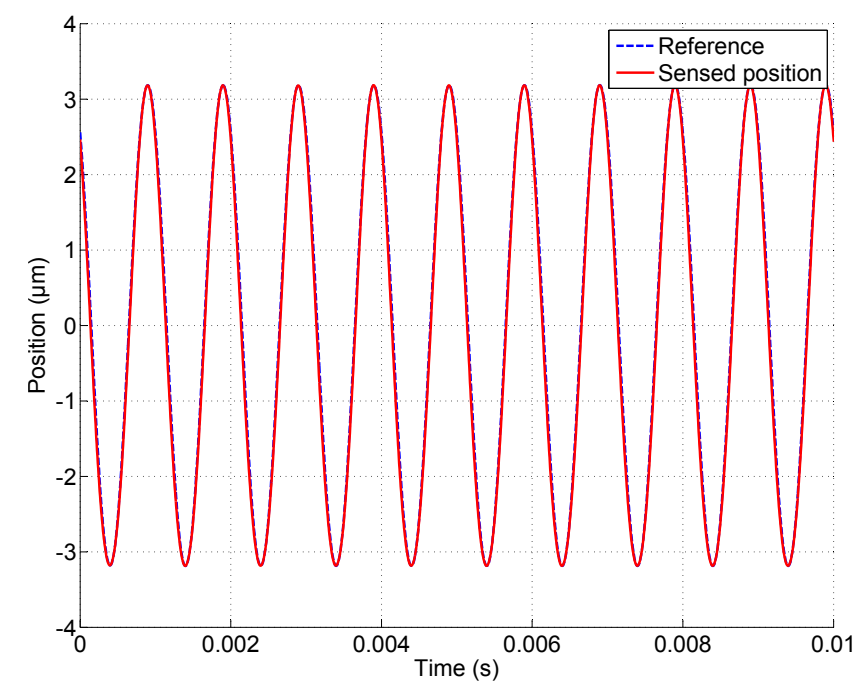

Fig. 7. Open-loop scan with frequency of $1000 \mathrm{~Hz}$ and range of $6 \mu \mathrm{m}$. The position was sensed using the GMR-based position sensor of the short-range scanner.

just two dominant vibrations modes in the frequency region up to $10 \mathrm{kHz}$. The effective masses, spring and damping constants are shown in Table I. Despite its conceptual and computational simplicity, the two-mass model provides an accurate estimate of the scanner dynamics. Experimental results presented later show that this estimate is also close to the real response of the scanner prototype.

\section{Displacement sensing}

The short-range scanner is equipped with a highly sensitive displacement sensor based on the effect of giant magnetoresistivity (GMR) [35], [13] for position measurements along the actuation axis. We have chosen a GMRbased sensor because of its high bandwidth, low level of measurement noise and small form factor. Besides meeting the above requirements on performance, GMR sensors have a low cost and require only simple read-out electronics.

The GMR sensing element used in the setup (NVE Corporation, Eden Prairie, MN, USA) has four sensing elements of size of approx. $40 \times 100 \mu \mathrm{m}$ connected in a Wheatstone bridge configuration. This configuration mitigates the effect of ambient temperature changes. The sensor is mounted on the stationary frame of the scanner under the scan table on which the sample is mounted. A permanent rare earth $(\mathrm{NdFeB})$ magnet of size $1.2 \mathrm{~mm} \times 2.7 \mathrm{~mm} \times 0.8 \mathrm{~mm}$ is mounted on the scan table such that the magnetic field at the GMR sensor is modulated as the scan table moves. The magnet is oriented such that its magnetic moment is in a direction perpendicular to the sensor plane and to the sensing direction. The position of the GMR sensor was fine-tuned to maximize the magnetic field gradient in the sensing direction that is acting on the GMR sensing elements; the standard deviation of the sensor noise over the frequency range of 0 to $10 \mathrm{kHz}$ is approx. $0.7 \mathrm{~nm}$. The non-linearity of the sensor over the effective sensing range depends on the range of motion, targeted sensitivity, the characteristics of the sensing element and the magnet geometry; the typical non-linearity
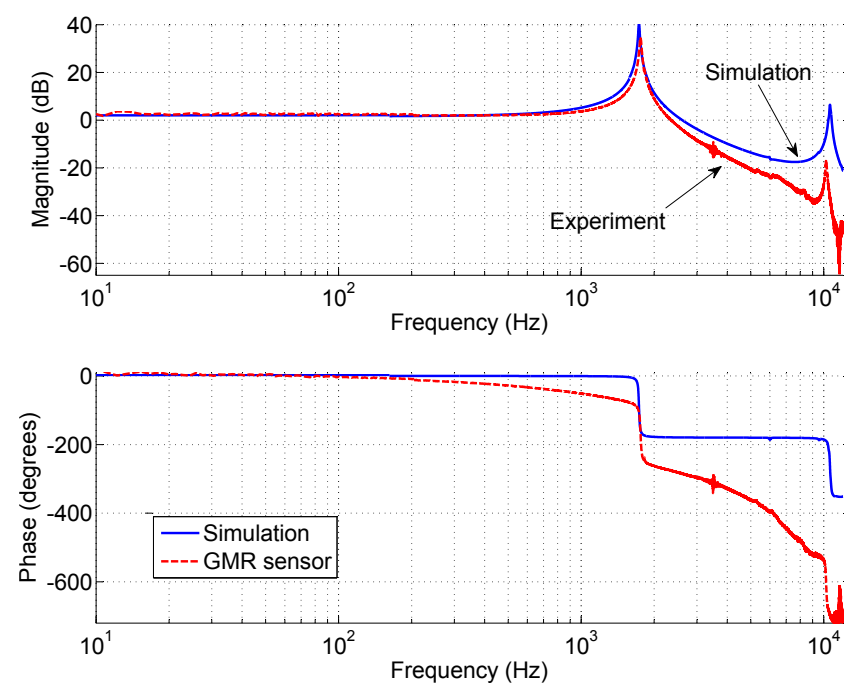

Fig. 8. Transfer function between the actuation signal and the scan table displacement in the actuation direction. The figure compares the frequency response obtained using finite-element simulations with the experimentally measured frequency response. The phase delay in the experimental measurement is due to the actuation amplifier.

measured was below $0.2 \%$. The sensor was calibrated against an externally mounted optical measurement probe (MTI Instruments MTI-2100).

\section{EXPERIMENTAL CHARACTERIZATION}

In this section, experimental characterization of a prototype of the short-range scanner designed in Section II is presented. The prototype was fabricated from a monolithic aluminum block using standard CAM processes. Measurements of the open-loop scan range and linearity, dynamic response and thermo-mechanical coupling measurements are presented.

\section{A. Scan range and linearity}

Figure 7 shows an experimentally measured open-loop response of the scanner to a harmonic actuation signal of $1000 \mathrm{~Hz}$ frequency which results in motion of approx. $6 \mu \mathrm{m}$ peak-to-peak range. The corresponding gain of the actuator is approx. $7.5 \mu \mathrm{m} / \mathrm{A}$. As expected from the electromagnetic actuator and the predicted dynamics of the scanner, the openloop scanner response is highly linear (with non-linearity of approx. $1.5 \%$ over the full scan range ) and has a sufficient range for short-range operation in the dual-stage system.

\section{B. Dynamic response}

The dynamics of the short-range scanner, predicted using finite element simulations, are shown in Figure 8 by a blue curve. The transfer function is computed between the applied actuator force (linear with drive current) and the displacement of the sample table. The frequency response of the fabricated prototype was obtained experimentally and is also shown in the figure, by a dashed red curve. There is a solid agreement between the dynamics as simulated and experimentally measured; the experimental results show a 


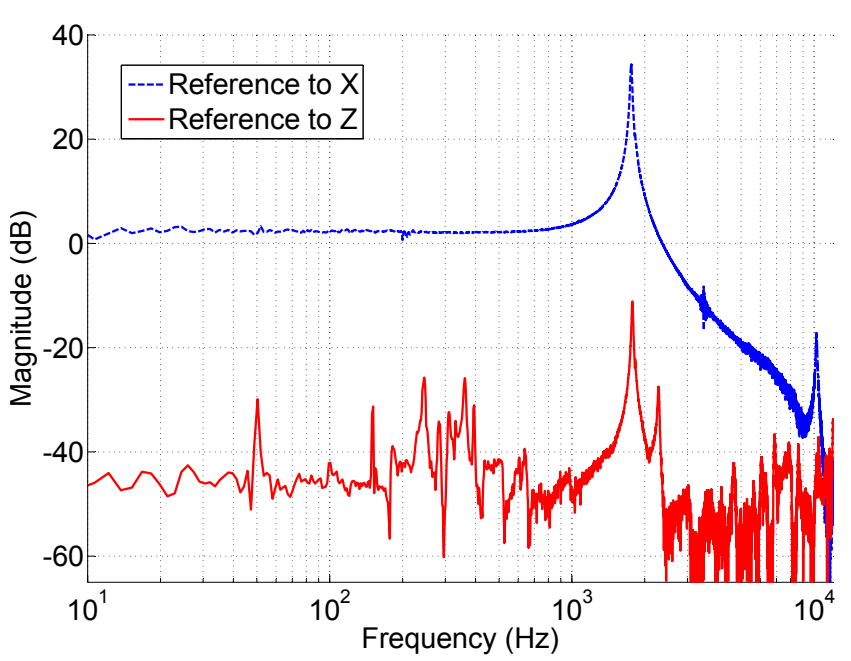

Fig. 9. Out-of-plane crosstalk measurement. The figure compares the experimentally obtained transfer function from the actuation signal to the displacement of the scan table in the actuation direction (denoted as $X$ ) with that to the out-of-plane scan table displacement (denoted as $Z$ ).

deviation in the first dominant resonant frequency of less than $1 \%$ with respect to the simulated results. This difference can occur due to uncertainties in the manufacturing process, variations in the materials used for fabrication, variations in the weight of the actuation components and loading of the scan table, for example due to the sample weight. Also, the finite-element model does not account for dynamics of the actuation and sensing components, such as the actuator amplifier. A slight variation in the dynamics is inevitable and can be compensated by means of feedback control.

As mentioned earlier, the connecting rod helps with mechanically attenuating the effects of any residual out-of-plane forces exercised by the actuator on the scan-table. With this feature, the crosstalk to the $y$ - and $z$-axis arising from the $x$ motion is very low; the attenuation of more than $80 \mathrm{~dB}$ and $50 \mathrm{~dB}$, respectively, is predicted in the simulations. The outof-plane cross-talk which was expected to be more dominant owing to the construction of the scanner was measured experimentally by using an externally mounted photonic sensor (MTI Instruments MTI-2100). Figure 9 compares the frequency response from the $x$ actuation signal to the $z$ motion signal with that to the intended $x$ motion signal. A direct experimental measurement of the frequency response between the $x$ actuation signal and the in-plane cross-talk to the $y$ axis was not possible due to the measurement probe inaccessibility; however, the imaging results presented later show that the $y$ cross talk is negligible.

\section{Thermo-mechanical coupling}

Passing electric current through the coil of the actuator generates heat. This effect is known as Joule or ohmic heating and is inevitable in electromagnetic actuators. According to Joule's first law, the power dissipated increases proportionally with the electrical resistance of the coil and with square of the current passed through the coil, i.e.

$$
P=I^{2} R
$$

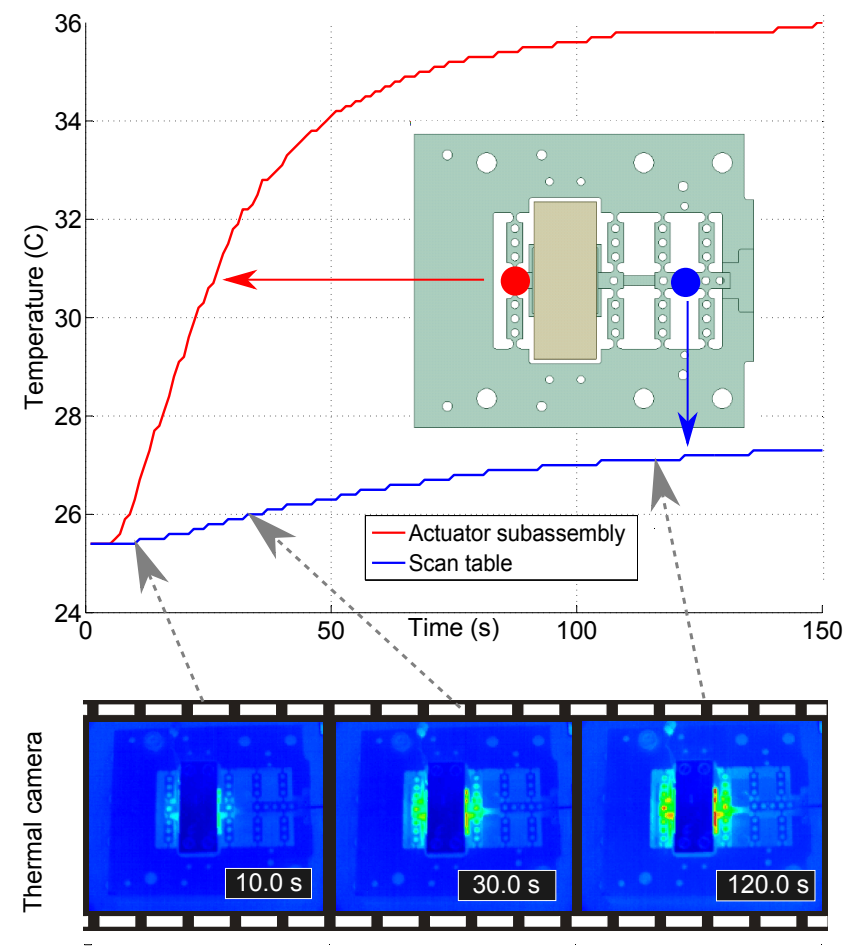

Fig. 10. Temperature distribution in the short-range scanner obtained by using an infra-red camera and calibrated temperature measurements using contact sensors positioned on the actuator subassembly and on the scan table, respectively. In the thermal camera image, the bright green color corresponds to a temperature increase of approx. $10^{\circ} \mathrm{C}$ with respect to the ambient room temperature (blue).

For example, with current $I=1 \mathrm{~A}$ and electrical resistance $R=5 \Omega$, the dissipated power will be approx. $P=5 \mathrm{~W}$. Hence, it is imperative that a sufficient thermal isolation is provided which will prevent heating of the scanner body and its unwanted thermal expansion.

In the short-range scanner, the effect of the actuator ohmic heating on the position of the scan table is significantly decreased due to the mechanical and thermal decoupling of the actuator sub-assembly and the scan table. Owing to the small-section connection rod, the scan table is thermally isolated from the actuator sub-assembly. The actuator subassembly also has a strong heat conduction pathway to the main body of the scanner.

The coil heating was investigated experimentally by mapping the temperature distribution of the scanner using an infra-red camera (FLIR SC5000) while the scanner is being actuated at its full scan range $(6 \mu \mathrm{m}$, corresponding current $0.8 \mathrm{~A}$ ). At the same time, point-based temperature measurements on the actuator subassembly and on the scan table were acquired using a calibrated thermometer (Fluke 542). The temperature profiles recorded and the corresponding time lapse image from the thermal camera are shown in Figure 10. The measurements clearly show that the scan table remains relatively cool in spite of a continuous operation of the scanner over 150 seconds. Whereas the actuator subassembly heats up by approximately $10^{\circ} \mathrm{C}$ due to the ohmic heating of the actuator coil, the increase of the scan table temperature is negligible over a short period time and does 


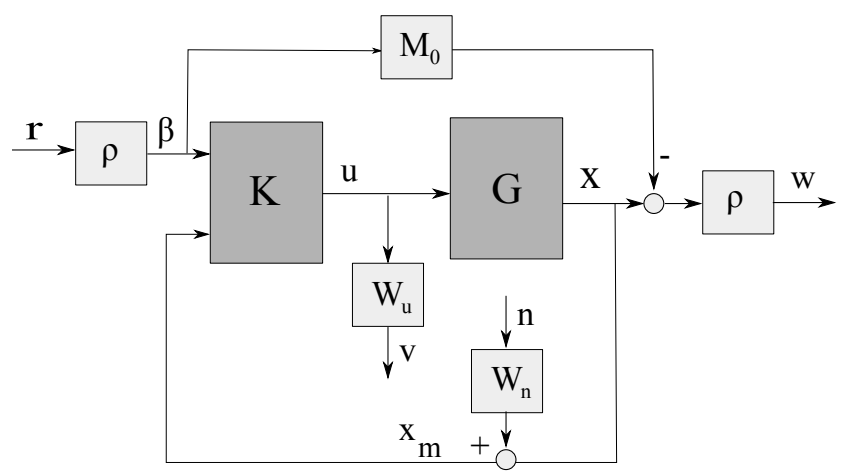

Fig. 11. A control design architecture for a two degree-of-freedom robust controller with direct shaping of the noise sensitivity transfer function.

not exceed $2{ }^{\circ} \mathrm{C}$ over 150 seconds. The resulting thermal expansion of the scan table in the transient period is approx. $600 \mathrm{~nm} /$ degree for the scan axis and $200 \mathrm{~nm} /$ degree for the out-of-plane axis, relative to the temperature of the actuator subassembly. For scan operations with a short duration, or scan operations performed in the steady-state period, the disturbance caused by the ohmic heating is significantly smaller; typically, the displacement fluctuations are below $100 \mathrm{~nm}$ and owing to their low bandwidth, can be readily compensated by means of feedback control.

\section{Feedback CONTROL}

To achieve high positioning accuracy at high speeds, a feedback controller is designed to control the motion of the short-range scanner based on the position information from the GMR sensor. A robust control design procedure for a two degree-of-freedom feedback controller is used in which the noise sensitivity of the feedback loop is directly shaped. The procedure combines the noise sensitivity shaping approach [15] with the robust performance design of [36] and is detailed in [32].

For purposes of the controller design, the dynamics of the short-range scanner were approximated with a second-order transfer function

$$
G(s)=g \cdot \frac{\omega_{n}^{2}}{s^{2}+2 \xi \omega_{n}+\omega_{n}^{2}} .
$$

The damping ratio $\xi$ and the natural frequency $\omega_{n}$ can be inferred from the mass-spring damper model introduced in Section II-C. Namely, the damping ratio is

$$
\xi=\frac{c_{a}+c_{s}}{2 \sqrt{\left(k_{a}+k_{s}\right)\left(m_{a}+m_{s}\right)}}
$$

and the resonant frequency is

$$
\omega_{n}=\frac{1}{2 \pi} \sqrt{\frac{k_{a}+k_{s}}{m_{a}+m_{s}}}
$$

With the model parameters introduced in Table I, the values used in the second order model are $\xi=0.005$ and $\omega_{n}=$ $1768 \mathrm{~Hz}$. As discussed in Section II-C, slight deviations in the actual resonant frequency and damping ratio can occur due to manufacturing uncertainties and the sample weight.

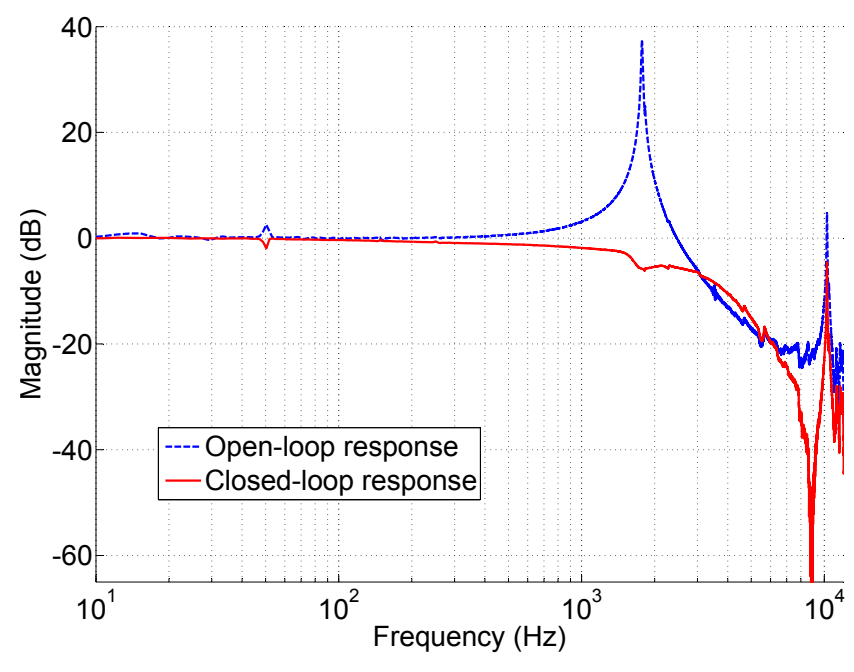

Fig. 12. Comparison of the open-looop and closed-loop transfer function of the scanner. The closed-loop frequency response is obtained using a two-degree of freedom noise-resilient feedback control design with approx. $40 \mathrm{pm}$ projected measurement noise.

The model in Equation 10 captures only the low frequency dynamics of the scanner and the first mechanical resonant mode at approx. $1.7 \mathrm{kHz}$. To keep the control design simple, the remaining resonant modes were omitted in the model and the resulting feedback loop was additionally shaped by a Butterworth band-stop filter centered at $10 \mathrm{kHz}$. The requirements on low sensitivity to the measurement noise, $n$, were reflected by choosing $W_{n}$ as a second-order transfer function with band-stop characteristics in the frequency range from $500 \mathrm{~Hz}$ to $7 \mathrm{kHz}$. The limitations on control effort, $u$, were expressed by choosing $W_{u}$ as a first-order low-pass filter with corner frequency of $10 \mathrm{kHz}$. In the process of control design, the targeted closed-loop transfer function $M_{0}$ between the reference, $r$, and the scanner position, $x$, and the scalar parameter $\rho$ were chosen such that a desired trade-off between robustness and performance was obtained. We refer the interested reader to [32] for more details on the control design.

The resulting controller has two degrees of freedom and consists of a prefilter component and a feedback component. The prefilter component is primarily responsible for matching the low frequency gain and partly compensates for the lightly damped pole at approx. $1.7 \mathrm{kHz}$. The feedback component has a very low gain at low frequencies and its control effort is focused into the frequency range of $1 \mathrm{kHz}$ to $7 \mathrm{kHz}$. This is important for achieving the required closedloop bandwidth and is in agreement with the limitations imposed by $W_{n}$. By design, there is no integral part in the controller; this is possible due to the linearity of the scanner. If needed, the integral part could be added by preshaping of the plant model prior to the control design.

The controller was discretized by using Tustin's method with sampling time of $20 \mu \mathrm{s}$ and implemented in a digital signal processor. Figure 12 compares the experimental openloop response of the plant with the experimental closed-loop response. The closed-loop response is flat with bandwidth of $1.5 \mathrm{kHz}$ which is important for tracking the broadband 


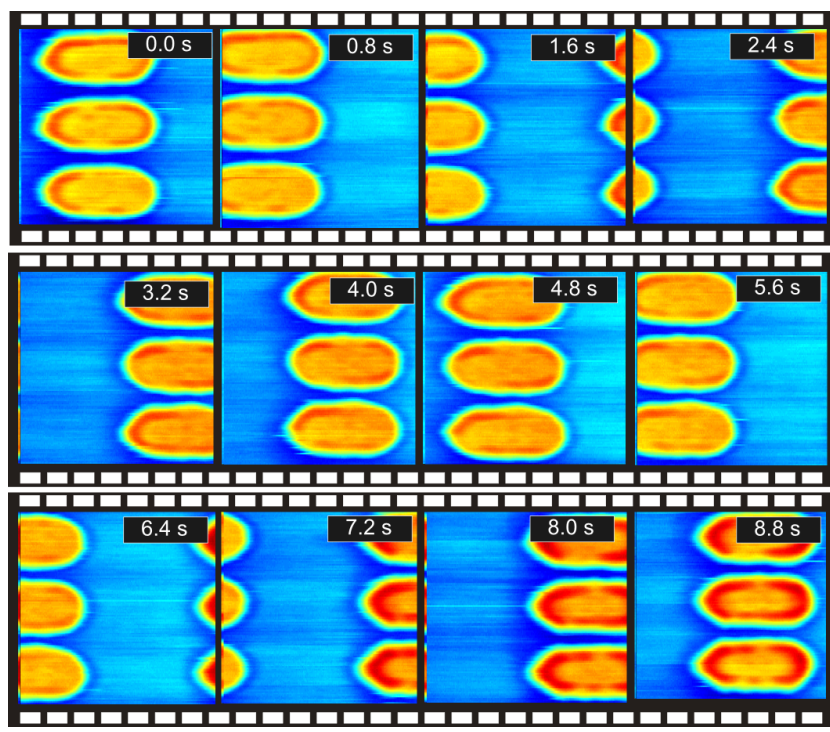

Fig. 13. A time-lapse sequence of high-resolution AFM topography estimates obtained in real-time by repeatedly shifting the sample along the $x$ scan direction and imaging the sample with 1.25 frames/s. The scan range is $2 \times 2 \mu \mathrm{m}$, the topographic height of the nanopatterns is approx. $100 \mathrm{~nm}$.

reference signals which arise in raster-based SPM. To evaluate the effect of the measurement noise which enters the feedback loop and affects the control action, we have used experimentally acquired noise measurements from the GMR sensor and computed the motion projected by the feedback loop to the output of the plant. It can be shown that the controller is sensitive to the measurement noise only in the frequency region of the resonant peak where feedback control is essential. Therefore, the standard deviation of the positioning error induced by the measurement noise in this feedback loop amounts to remarkable $0.04 \mathrm{~nm}$. Note that this is the positioning error arising from measurement noise alone and that there might be other sources of positioning error such as the actuator and amplifier noise.

For a good overall performance, it is essential that all three axes of the large-range positioner are under feedback control. To that end, we used the standard Physik Instrumente PIE612.C0 control box with analog integral controllers which use the built-in capacitive position sensors of the PI-517 stage. The controllers were tuned to approx. $20 \mathrm{~Hz}$ bandwidth which was sufficient for the low-bandwidth large-range scan operation. The cross-coupling between the large-range scanner and the short-range scanner was experimentally measured to be below $-30 \mathrm{~dB}$ and did not require additional active compensation.

\section{AFM EXPERIMENTS}

This section presents experiments in which the dual-stage nanopositioning system is used for imaging in a customdesigned high-speed AFM setup. The imaging experiments are based on the dual-stage raster scanning process. In this process, the sample is traversed back and forth in the $x$ axis by driving the short-range scanner with a high-frequency triangular waveform. The $y$-axis of the large-range scanner shifts the sample continuously in the direction orthogonal

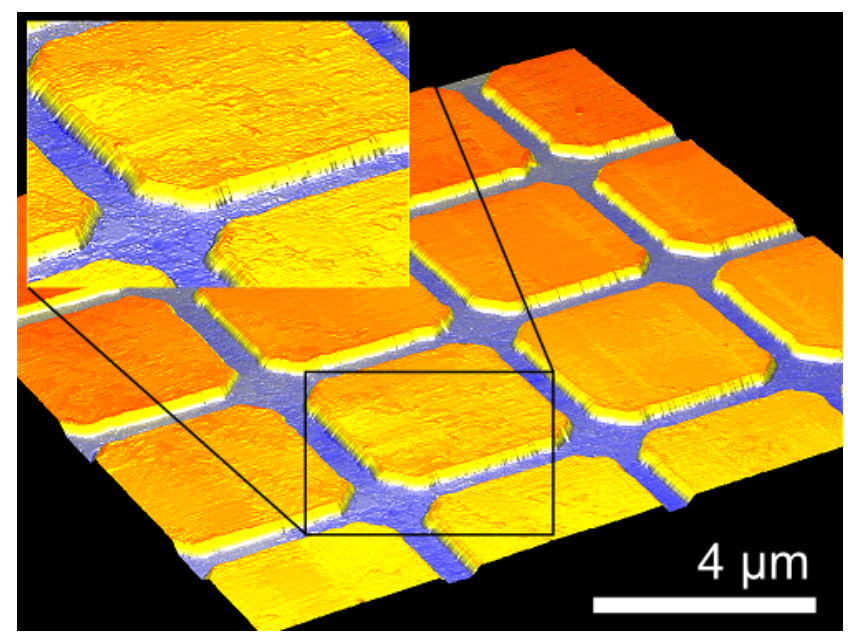

Fig. 14. A three-dimensional topography reconstruction of the lithographically patterned silicon surface. The image is based on the combined data from an array of 64 subimages acquired by the dual-stage nanopositioning system.

to the fast scan motion while the $x$-axis of the largerange scanner provides a DC offset to the fast scan motion. The $z$-axis of the large-range scanner is used to engage the cantilever with the sample surface during the imaging process.

Two experiments are presented: in the first experiment, the dual-stage system is used for high-speed short-range imaging with frame rates of more than 1 frame/s. In the second experiment, a comparably large sample area is decomposed into an array of small-range images and assembled into a single high-resolution image. In both experiments, the shortrange and large-range scanner are under feedback control as described in Section IV. To allow for high precision tracking, the actuation frequencies are chosen such that at least the first three harmonics of the actuation signal are within the closedloop bandwidth. Alternatively, scan trajectories with a more intricate shape could be used [37], [38], [39].

In the imaging process, we used cantilevers with thermoelectric sensing capability [40], [41] and operated them in constant-height mode. The samples consist of a silicon surface with lithographic patterns of different dimensions; for each experiment, a different sample was used.

\section{A. High-frame-rate imaging}

In the first experiment, the short-range scanner was actuated with a triangular waveform reference signal of frequency $250 \mathrm{~Hz}$. The scan range was $2 \mu \mathrm{m} \times 2 \mu \mathrm{m}$, with spatial resolution $3 \mathrm{~nm}$ in the $x$-scan direction and $10 \mathrm{~nm}$ in the $y$-scan direction. Hence, a single frame consists of $655 \times$ 200 pixels, and its imaging is completed in $0.8 \mathrm{~s}$, yielding a frame rate of more than 1 frame/s. With this frame rate, interactive AFM imaging can be performed, in which highquality AFM images are obtained on-the-fly.

During the experiment, the sample under investigation was examined along the $x$-scan direction. First, an AFM image was obtained at a given sample location, and subsequently, the position of the sample relative to the probe was shifted 
along the $x$-scan direction by using the large-range scanner. Figure 13 shows time-lapse video images that was obtained during the experiment in which twelve images with a spacing of $400 \mathrm{~nm}$ were taken. Owing to the high-speed capabilities of the short-range scanner, real-time imaging of the sample was possible while the large-range scanner enabled largerange navigation over the sample surface.

\section{B. Large-range imaging}

In the second experiment, the imaged area of $16 \mu \mathrm{m} \times 16$ $\mu \mathrm{m}$ was divided into 64 regular subregions of $2 \mu \mathrm{m} \times 2 \mu \mathrm{m}$ which were imaged separately and patched together to form the resulting image. Each of the subframes was acquired in approx. $1.8 \mathrm{~s}$ by driving the short-range scanner with a triangular waveform of $113 \mathrm{~Hz}$ frequency, with the whole image being completed in approx. two minutes. The spatial resolution is $10 \mathrm{~nm}$. Owing to the excellent mechanical properties of the short-range scanner, precise calibration of the sensors and the use of feedback control, the subframes are almost perfectly aligned and constitute a high-resolution, high-quality image of more than 2.5 million pixels. The 3D topography reconstruction based on the combined image data is shown in Figure 14.

\section{CONCLUSION}

We presented a dual-stage approach to high-speed nanopositioning in which the conventional design trade-offs between scanner speed, range and closed-loop resolution are addressed by combining a low-speed, large-range scanner with a high-speed, short-range scanner. We presented the design, finite-element simulations, modeling and experimental characterization of an electromagnetically actuated shortrange scanner. Using a magnetoresistance-based sensor and a tailored noise-resilient feedback control design, we showed that the scanner can be operated in closed loop with a positioning resolution of $0.04 \mathrm{~nm}$. With the fast axis closed-loop bandwidth of $1.5 \mathrm{kHz}$ the dual-stage system designed in this paper was used in a custom-built atomic force microscope to image an area of $2 \times 2 \mu \mathrm{m}$ with resolution of $655 \times$ 200 pixels at a scan rate of 1.25 frames/s; using the same system, a 2.5 megapixel image of an area of $16 \times 16 \mu \mathrm{m}$ was obtained in approx. 2 minutes.

\section{ACKNOWLEDGMENTS}

The authors gratefully acknowledge Urs Egger for his assistance with the electronic hardware used in the experiments and Heiko Wolf for providing the sample. We thank Giorgio Signorello for his help with the characterization of the sample and Charlotte Bolliger for her assistance with the preparation of the manuscript. Special thanks go to Haris Pozidis and Evangelos Eleftheriou for their support of this work.

\section{REFERENCES}

[1] E. Eleftheriou, T. Antonakopoulos, G. Binnig, G. Cherubini, M. Despont, A. Dholakia, U. Durig, M. Lantz, H. Pozidis, H. Rothuizen, and P. Vettiger, "Millipede - a MEMS-based scanning-probe data-storage system," IEEE Transactions on Magnetics, vol. 39, no. 2, pp. 938 945, Mar. 2003.
[2] R. A. Oliver, "Advances in AFM for the electrical characterization of semiconductors," Reports on Progress in Physics, vol. 71, no. 7, p. 076501, 2008.

[3] T. Ando, T. Uchihashi, and T. Fukuma, "High-speed atomic force microscopy for nano-visualization of dynamic biomolecular processes," Progress in Surface Science, vol. 83, pp. 337-437, 2008.

[4] S. M. Salapaka and M. V. Salapaka, "Scanning probe microscopy," IEEE Control Systems Magazine, vol. 28, no. 2, pp. 65 - 83, 2008.

[5] S. Devasia, E. Eleftheriou, and S. O. R. Moheimani, "A survey of control issues in nanopositioning," IEEE Transactions on Control Systems Technology, vol. 15(5), pp. 802-823, 2007.

[6] G. Binnig and D. Smith, "Single-tube three-dimensional scanner for scanning tunneling microscopy," Review of Scientific Instruments, vol. 57 , no. 8 , pp. $1688-1689,1986$.

[7] J. Shim and D. Gweon, "Piezo-driven metrological multiaxis nanopositioner," Review of Scientific Instruments, vol. 72, p. 4183, 2001.

[8] S. Salapaka, A. Sebastian, J. Cleveland, and M. Salapaka, "High bandwidth nano-positioner: A robust control approach," Review of scientific instruments, vol. 73, p. 3232, 2002.

[9] G. Schitter and A. Stemmer, "Identification and open-loop tracking control of a piezoelectric tube scanner for high-speed scanning-probe microscopy," IEEE Transactions on Control Systems Technology, vol. 12, no. 3, pp. 449-454, 2004.

[10] G. Schitter, K. J. Astrom, B. E. DeMartini, P. J. Thurner, K. L. Turner, and P. K. Hansma, "Design and modeling of a high-speed AFMScanner," IEEE Transactions on Control Systems Technology, vol. 15 (5), pp. $906-915,2007$

[11] K. Leang and A. Fleming, "High-speed serial-kinematic SPM scanner: design and drive considerations," Asian journal of control, vol. 11, no. 2, pp. 144-153, 2009.

[12] B. J. Kenton and K. K. Leang, "Design and control of a threeaxis serial-kinematic high-bandwidth nanopositioner," IEEE/ASME Transactions on Mechatronics, vol. 17, no. 2, pp. $356-369$, april 2012.

[13] V. Kartik, A. Sebastian, T. Tuma, A. Pantazi, H. Pozidis, and D. Sahoo, "High-bandwidth nanopositioner with magnetoresistance based position sensing," Mechatronics, 2011

[14] S. O. R. Moheimani and B. J. G. Vautier, "Resonant control of structural vibration using charge-driven piezoelectric actuators," IEEE Transactions on Control Systems Technology, vol. 13, no. 6, pp. 1021$1035,2005$.

[15] A. Sebastian, A. Pantazi, S. O. R. Moheimani, H. Pozidis, and E. Eleftheriou, "Achieving subnanometer precision in a MEMS-based storage device during self-servo write process," IEEE Transactions on Nanotechnology, vol. 7, no. 5, pp. 586 - 595, 2008

[16] A. Sebastian and S. O. R. Moheimani, "Signal transformation approach to fast nanopositioning," Review of scientific instruments, vol. 80, no. 7, pp. 076101-1 - 076101-3, 2009.

[17] T. Tuma, A. Sebastian, W. Häberle, J. Lygeros, and A. Pantazi, "Impulsive control for fast nanopositioning," Nanotechnology, vol. 22, p. 135501, 2011.

[18] D. Croft, G. Shed, and S. Devasia, "Creep, hysteresis, and vibration compensation for piezoactuators: atomic force microscopy application." Journal of Dynamic Systems, Measurement, and Control(Transactions of the ASME), vol. 123, no. 1, pp. 35-43, 2001.

[19] B. Chen, T. Lee, K. Peng, and V. Venkataramanan, Hard disk drive servo systems. Springer Publishing Company, Incorporated, 2010.

[20] M. A. Lantz, H. E. Rothuizen, U. Drechsler, W. Haeberle, and M. Despont, "A vibration resistant nanopositioner for mobile parallel-probe storage applications," Journal of Microelectromechanical Systems, vol. 16 , no. 1 , pp. $130-139,2007$.

[21] A. Pantazi, A. Sebastian, T. A. Antonakopoulos, P. Baechtold, A. R. Bonaccio, J. Bonan, G. Cherubini, M. Despont, R. A. DiPietro, U. Drechsler, U. Duerig, B. Gotsmann, W. Haeberle, C. Hagleitner, J. L. Hedrick, D. Jubin, A. Knoll, M. A. Lantz, J. Pentarakis, H. Pozidis, R. C. Pratt, H. Rothuizen, R. Stutz, M. Varsamou, D. Wiesmann, and E. Eleftheriou, "Probe-based ultrahigh-density storage technology," IBM Journal of Research and Development, vol. 52, no. 4.5, pp. $493-511,2008$.

[22] G. Cherubini, R. Cideciyan, L. Dellmann, E. Eleftheriou, W. Haeberle, J. Jelitto, V. Kartik, M. Lantz, S. ÖLCER, A. Pantazi et al., "29.5-gb/in2 recording areal density on barium ferrite tape," IEEE transactions on Magnetics, vol. 47, no. 1, pp. 137-147, 2011.

[23] H. Butler, "Position control in lithographic equipment [applications of control]," Control Systems, IEEE, vol. 31, no. 5, pp. 28 -47, oct. 2011.

[24] S. G. Stan, The CD-ROM drive: A brief system description. Kluwer Academic Publishers, 1998. 
[25] M. Kobayashi and R. Horowitz, "Track seek control for hard disk dual-stage servo systems," Magnetics, IEEE Transactions on, vol. 37, no. 2, pp. 949-954, 2001.

[26] S. Kwon, W. K. Chung, and Y. Youm, "On the coarse/fine dual-stage manipulators with robust perturbation compensator," in Proceedings of IEEE International Conference on Robotics and Automation, vol. 1. IEEE, 2001, pp. 121-126.

[27] J. E. McInroy and S. S. Aphale, "Optimal filters from task velocities to joint velocities including both position and velocity limits," in Proceedings of the 42nd IEEE Conference on Decision and Control, vol. 6. IEEE, 2003, pp. 5885-5890.

[28] Q. Xu, "Design and development of a flexure-based dual-stage nanopositioning system with minimum interference behavior," IEEE Transactions on Automation Science and Engineering, vol. 9, no. 3, pp. 554-563, 2012.

[29] G. Schitter, W. F. Rijkee, and N. Phan, "Dual actuation for highbandwidth nanopositioning," Proceedings of the IEEE Conference on Decision and Control, pp. 5176-5181, 2008.

[30] S. Kuiper and G. Schitter, "Model-based feedback controller design for dual actuated atomic force microscopy," Mechatronics, vol. 22, no. 3, pp. 327-337, 2012.

[31] S. Andersson, "Curve tracking for rapid imaging in afm," IEEE Transactions on NanoBioscience, vol. 6, no. 4, pp. 354-361, 2007.

[32] T. Tuma, W. Haeberle, H. Rothuizen, J. Lygeros, A. Pantazi, and A. Sebastian, "A dual-stage nanopositioning approach to high-speed scanning probe microscopy," Proceedings of the 2012 Conference on Decision and Control, pp. 5079 - 5084, 2012.

[33] — - "A high-speed electromagnetically-actuated sanner for dualstage nanopositioning," Proceedings of the 6th IFAC Symposium on Mechatronic Systems, 2013, to appear.

[34] R. Yang, M. Jouaneh, and R. Schweizer, "Design and characterization of a low-profile micropositioning stage," Precision Engineering, vol. 18, no. 1, pp. 20-29, 1996.

[35] S. Wolf, D. Awschalom, R. Buhrman, J. Daughton, S. Von Molnar, M. Roukes, A. Chtchelkanova, and D. Treger, "Spintronics: A spinbased electronics vision for the future," Science, vol. 294, no. 5546, pp. 1488-1495, 2001.

[36] D. Limebeer, E. Kasenally, and J. Perkins, "On the design of robust two degree of freedom controllers," Automatica, vol. 29, no. 1, pp. 157-168, 1993.

[37] A. Fleming and A. Wills, "Optimal periodic trajectories for bandlimited systems," IEEE Transactions on Control Systems Technology, vol. 17, no. 3, pp. 552-562, 2009.

[38] T. Tuma, J. Lygeros, A. Sebastian, and A. Pantazi, "Optimal scan trajectories for high speed scanning probe microscopy," Proceedings of the 2012 American Control Conference, pp. 3791-3796, 2012.

[39] T. Tuma, J. Lygeros, V. Kartik, A. Sebastian, and A. Pantazi, "Highspeed multiresolution scanning probe microscopy based on Lissajous scan trajectories," Nanotechnology, vol. 23, p. 185501, 2012.

[40] W. P. King, T. W. Kenny, K. E. Goodson, G. Cross, M. Despont, U. Dürig, H. Rothuizen, G. Binnig, and P. Vettiger, "Atomic force microscope cantilevers for combined thermomechanical data writing and reading," Applied Physics Letters, vol. 78, pp. 1300-1302, 2001.

[41] A. Sebastian and D. Wiesmann, "Modeling and experimental identification of silicon microheater dynamics: A systems approach," Journal of Microelectromechanical Systems, vol. 17, no. 4, pp. 911-920, 2008. 